\title{
Avenir et climat : représentations de l'avenir dans des blogs francophones portant sur le changement climatique
}

Future and climate: representations of the future in French language blogs related to climate change.

Porvenir y clima: representaciones del futuro en blogs francófonos sobre el tema del cambio climático

Kjersti Fløttum, Øyvind Gjerstad et Anje Müller Gjesdal

\section{OpenEdition}

Journals

Édition électronique

URL : https://journals.openedition.org/mots/24270

DOI : $10.4000 /$ mots. 24270

ISSN : 1960-6001

Éditeur

ENS Éditions

Édition imprimée

Date de publication : 5 mars 2019

Pagination : 33-50

ISBN : 979-10-362-0130-1

ISSN : 0243-6450

\section{Référence électronique}

Kjersti Fløttum, Øyvind Gjerstad et Anje Müller Gjesdal, « Avenir et climat : représentations de l'avenir dans des blogs francophones portant sur le changement climatique ", Mots. Les langages du politique [En ligne], 119 | 2019, mis en ligne le 01 janvier 2022, consulté le 23 avril 2022. URL : http://

journals.openedition.org/mots/24270; DOI : https://doi.org/10.4000/mots.24270 


\section{Avenir et climat : représentations de l'avenir dans des blogs francophones portant sur le changement climatique*}

Le but du présent article est d'explorer la manière dont l'avenir est perçu, c'està-dire les diverses valences de cette perception, dans les blogs portant sur le changement climatique. Le défi du climat est au cœur des discours écologiques contemporains, et différentes études ont montré que la communication sur les effets du changement climatique est intimement liée aux mesures politiques proposées et aux projets de société envisagés (Hulme, 2009; Moser, 2016 ; Painter, 2013; Pearce et al., 2015). Les différents discours (scientifique, politique, des militants, des citoyens) créent, reproduisent, mettent en question et excluent diverses représentations du monde, qui sont au fondement de décisions, de contestations et d'actions (Fløttum, 2016; Fløttum éd., 2017). De ce point de vue, l'étude des représentations de l'avenir en lien avec le contexte climatique et environnemental nous permet de révéler les prémisses des priorités politiques liées aux changements du climat.

Bien que la question de l'avenir soit abordée dans de nombreux débats, tant publics que scientifiques (par exemple dans les scénarios des rapports les plus récents publiés par le GIEC1), les représentations linguistiques de l'avenir restent encore peu examinées. Nous nous proposons de les examiner dans un large corpus de blogs francophones liés au changement climatique, le NTAP French corpus (Salway et al., 2016), en enquêtant sur les deux mots avenir et futur. Notre objectif principal est la recension des perspectives optimistes (d'une société durable) et des perspectives pessimistes (de conséquences dramatiques), mais aussi leur mise en regard. Nous focaliserons l'analyse sur les

* Nous tenons à remercier les éditeurs et les trois relecteurs anonymes pour leurs évaluations pertinentes, qui ont contribué à améliorer l'article de manière décisive.

1. Groupe d'experts intergouvernemental sur l'évolution du climat (GIEC).

Université de Bergen kjersti.flottum@uib.no

Université de Bergen

oyvind.gjerstad@uib.no

Centre d'études supérieures de Østfold

anje.m.gjesdal@hiof.no 
blogs des années 2009-2010 (période entourant la COP 15 de Copenhague) et les années 2013-2014 (période de la publication des rapports AR5 du GIEC). L'approche analytique sera principalement qualitative, dans une perspective lexicale basée sur une analyse de contenu et centrée sur le langage évaluatif. Elle vise à donner un nouvel éclairage aux conceptions écologiques de l'avenir.

L'étude de représentations de l'avenir subsume quatre questions que nous formulerons comme suit :

1. Comment les perspectives positives et optimistes (d'une société durable), opposées aux perspectives négatives et pessimistes (de la « fin du monde»), sont-elles évoquées à travers les différentes occurrences d'avenir et futur?

2. Comment se caractérisent les expressions évaluatives dans lesquelles avenir et futur peuvent être insérés?

3. Que suppose la notion d'avenir, lorsque celle-ci est évoquée à travers les termes avenir et futur?

4. Peut-on y lire une évolution de la perception de l'avenir entre les années 2009-2010 (période de la conférence COP 15 de Copenhague) et les années 2013-2014 (période de la publication des rapports AR5 du GIEC)?

Après une brève revue de la question, nous exposerons nos matériaux et méthodes, puis les résultats de nos analyses. Nous terminerons cet article par une discussion de nos résultats.

\section{État de l'art}

Le changement climatique et les questions écologiques ont été largement abordés sous différents angles, notamment par la science politique, la sociologie et les sciences de l'information et de la communication (Comby, 2015; Grundmann, Krishnamurthy, 2010 ; Schäfer, Schlichting, 2014; Tvinnereim, Fløttum, 2015; Zaccai et al. éd., 2012). Les approches discursives et linguistiques, tournées vers les représentations, sont également de plus en plus nombreuses (pour un aperçu, voir par exemple Fløttum, 2016). Ces études se fondent sur des matériaux différents, à savoir des rapports scientifiques, des documents politiques, des textes journalistiques, divers exemples de médias sociaux et de blogs, des discours d'enquête (Fløttum éd., 2017; Fløttum, Dahl, 2012, 2014 ; Fløttum, Gjerstad, 2016; Gjesdal, Gjerstad, 2015; Grundmann, Krishnamurthy, 2010; Koteyko, 2010; Tvinnereim et al., 2017).

Les phénomènes climatiques et écologiques ont donc été étudiés dans diverses perspectives linguistiques et langagières. Cependant, à notre connaissance, la notion d'avenir n'a pas encore fait l'objet d'études approfondies dans un contexte francophone. Notre but est de combler cette lacune, tout en nous inspirant d'une étude faite sur le mot anglais future dans un corpus de blogs 
anglophones liés aux questions de climat (Fløttum et al., 2014). Dans une approche quantitative et qualitative, les auteurs de cet article proposent une série de catégories caractérisant les occurrences et contextes du mot future. Ils ont observé une fréquence notable de caractérisations liées à la notion de «sustainability» («développement durable») ainsi qu'une présence importante d'expressions à valence positive. Ils ont également noté que le changement climatique était considéré comme une menace (surtout pour la nature), mais aussi comme une opportunité (notamment pour la vie économique). Enfin, ils postulent la polarisation des perspectives du « gloom-and-doom » jusqu'au «bright future ( ibid., p. 213). Bien que les méthodes ainsi que les questions de recherche de cette étude se distinguent de nos méthodes et questions, on pourra comparer, dans une certaine mesure, les résultats. Nous y reviendrons dans la dernière partie.

Signalons également le travail de Geneviève Petiot (1994), qui, à travers l'étude d'un corpus de livres de vulgarisation écrits par des spécialistes et d'ouvrages d'une large diffusion, retrace l'entrée du mot écologie en politique. L'auteure observe que le transfert du mot du domaine scientifique à celui de la politique s'opère relativement rapidement au mitan des années 1970, et que ce déplacement lexical a donné au mot écologie « [s]es contours sémantiques et valeurs discursives» (ibid. p. 71, 76). Cette auteure remarque en outre que ces ouvrages ont tendance à souligner les risques et les menaces associés à la question de l'environnement :

Une constante dans tous les ouvrages, tant de vulgarisation scientifique que de diffusion large : c'est le thème de la menace, du risque, toujours présent. [...] Le corpus abonde en termes plus ou moins spécifiques. On passe de la pollution, omniprésente, il serait plus juste de parler des pollutions, les domaines concernés étant précisés, manifestées notamment par les déchets produits par l'activité nocive de l'homme. Défilent les problèmes de l'eau, du gaz carbonique, de l'ozone, de la déforestation, des pesticides, des pluies acides et bien sûr du nucléaire, sans oublier les fameux CFC. L'effet de serre est alors évoqué, voire discuté, les mots de risque(s), danger(s), et même mort affluent et les solutions sont recherchées. (lbid., p. 74 ; les italiques sont de l'auteur)

D’autres auteurs observent la tendance à la conflictualité des discours environnementaux, et plus spécifiquement des discours portant sur le changement climatique. Dans une étude des polémiques climatiques telles qu'elles sont présentées par les climatologues d'une part et la presse française d'autre part, Marion Mauger-Parat et Ana Carolina Peliz (2013) observent que les experts jouent un rôle dans ladite conflictualité. Le changement climatique n'étant en effet pas directement observable par le grand public, la mobilisation s'est constituée à partir de l'expertise scientifique, notamment relayée par le GIEC, pour devenir un problème politique et un sujet de débats intenses dans la presse et sur internet (Mauger-Parat, Peliz, 2013). 
Pour résumer, des études antérieures indiquent que les discours environnementaux en général et les débats sur le changement climatique en particulier se caractérisent a) par la conflictualité et b) par la présence d'un vocabulaire dysphorique. Nous questionnerons donc la manière dont ces tendances s'expriment dans la façon de représenter et d'envisager l'avenir dans les discours sur le changement climatique.

\section{Matériaux et méthodes}

Nos données sont tirées du corpus NTAP French corpus, disponible via la plateforme Corpuscle/Clarino à l'université de Bergen (Meurer, 2012). Ce corpus comporte des billets de 2033 blogs francophones, portant sur le changement climatique et couvrant les années 1974-2014, soit 1506074082 mots au total. Le corpus a été construit selon une approche thématique («topically-focused ») : les blogs ont été sélectionnés en fonction de l'occurrence d'un lexique lié au changement climatique. Le corpus est donc spécifique au domaine du changement climatique dans un sens large ${ }^{2}$.

Afin de sélectionner nos données, nous avons, dans un premier temps, identifié les collocations les plus importantes du corpus, en utilisant l'outil statistique intégré dans Corpuscle. Cet outil associe la mesure de l'information mutuelle avec une mesure de fréquence. Cette analyse a donné 4413 collocations associées au mot pivot avenir. Ensuite, nous avons focalisé l'analyse sur les formes avenir + adjectif, en excluant les adjectifs exprimant la temporalité (proche, immédiat). De cette recherche nous avons obtenu les dix collocations les plus fréquentes, exposées dans la liste suivante :

1. avenir radieux

2. avenir incertain

3. avenir prometteur

4. avenir meilleur

5. bel avenir

6. avenir prévisible

7. sombre avenir

8. avenir i-radieux

9. avenir énergétique

10. avenir bouché

Si l'on se fonde sur le seul langage évaluatif ou la polarité positif-négatif (Kerbrat-Orecchioni, 2009), cette liste montre une perception hétérogène du changement climatique. Or, une analyse des concordances (outil de présentation d'extraits de texte contenant un mot spécifique; Anthony, 2005) montre

2. Pour plus de détails, voir http://clarino.uib.no/korpuskel/page (consulté le 08/11/2018). 
l'insuffisance d'une approche par collocations (combinaisons de mots privilégiées). Prenons l'exemple d'avenir radieux, première forme de la liste avec 985 occurrences dans le corpus et qui relève a priori d'une conceptualisation positive de l'avenir. En effet, l'analyse des concordances indique que le syntagme avenir radieux relève souvent d'un emploi ironique, où le point de vue est attribué à d'autres acteurs et non pas au blogueur, comme le montre l'exemple suivant :

L'avenir du transport aérien dans un contexte de pic du pétrole et de montée inéluctable de politiques de réduction des émissions de gaz à effet de serre est sombre, ce que veulent ignorer les avocats du projet, qui croient à l'avenir radieux du low cost (des champions du travail low quality) en fermant les yeux sur ces arguments. $(\mathrm{A}-\mathrm{C}-13-14)^{3}$

En outre, l'approche par collocations s'avère insuffisante pour une seconde raison. En effet, un examen des concordances montre que les collocations ne sont pas nécessairement limitées au domaine du changement climatique.

Nous avons donc effectué de nouvelles recherches, pour cibler les mots clés avenir et futur. Nous avons obtenu un total de 200920 occurrences d'avenir et 87668 de futur. En considérant de plus près un échantillon de ces données, nous avons à nouveau observé qu'une bonne partie des occurrences n'était pas directement liée à la question du climat ou de l'écologie. Nous avons donc procédé à des recherches plus ciblées quantitativement, mais aussi qualitativement, pour identifier les énoncés pertinents. Après différents essais, nous avons opté pour la combinaison entre les mots avenir et futur avec les mots climatique et réchauffement, en permettant une distance entre les mots recherchés de 1 à 20 mots. Voici un exemple de la formule de requête utilisée :

[réchauffement $\{1,20\}$ avenir], suivie de l'indication des années visées.

Il en est résulté 16 recherches, qui ont donné au total 711 énoncés avec les occurrences des mots en question, réparties comme suit, en fonction des différentes combinaisons entre avenir, futur, réchauffement et climatique et selon les deux périodes 2009-2010 et 2013-2014 (avec le nombre d'occurrences mis entre parenthèses) :
1. réchauffement-avenir 2009-2010 (54)
2. avenir - réchauffement 2009-2010 (41)
3. climatique - avenir 2009-2010 (57)
4. avenir - climatique 2009-2010 (38)
5. réchauffement - futur 2009-2010 (22)
6. futur-réchauffement 2009-2010 (44)

3. Les abréviations renvoient au corpus de la manière suivante $: R=$ réchauffement; $A=$ avenir; $\mathrm{C}=$ climatique $; \mathrm{F}=$ futur $; 09-10=$ la période 2009-2010; 13-14 = la période 2013-2014. Les extraits de blogs sont reproduits dans leur orthographe, leur ponctuation et leur typographie originales. 
7. climatique - futur 2009-2010 (52)

8. futur-climatique 2009-2010 (70)

9. réchauffement-avenir 2013-2014 (36)

10. avenir - réchauffement 2013-2014 (22)

11. climatique - avenir 2013-2014 (68)

12. avenir - climatique 2013-2014 (42)

13. réchauffement-futur 2013-2014 (31)

14. futur - réchauffement 2013-2014 (35)

15. climatique - futur 2013-2014 (49)

16. futur - climatique 2013-2014 (50)

Nous avons ainsi obtenu 378 occurrences pour la période 2009-2010 et 333 occurrences pour la période 2013-2014.

On constate qu'il n'y a pas de très grandes différences de fréquence entre les recherches, celles-ci produisant des résultats allant de 22 à 68 occurrences. Cependant, il se peut que ces différences soient à imputer à l'ordre des mots et aux choix syntaxiques. C'est là une perspective intéressante que nous n'avons pas pu poursuivre dans le cadre du présent travail4. Soulignons qu'aucun blog ne domine dans la période 2009-2010, et qu'il est rare de trouver plus de cinq occurrences relevant d'un même blog. En revanche, durant la deuxième période (2013-2014), une grande partie des occurrences (entre 10 et $40 \%$, selon les paramètres de la recherche) sont tirées du blog «enjeuxenergies.wordpress. com » de l'Institut de recherche en architecture sociale durable.

Après avoir collecté les occurrences pertinentes, nous avons effectué une analyse qualitative de toutes ces occurrences selon le schéma suivant :

a. la représentation de l'avenir dans une perspective positive, négative, neutre ou de refus de la problématique climatique;

b. la présence de lexèmes évaluatifs/axiologiques, c'est-à-dire qui sont porteurs d'un «jugement évaluatif, d'appréciation ou de dépréciation» (Kerbrat-Orecchioni, 2009, p. 83), éventuellement la présence de lexèmes auxquels le contexte permet de donner une interprétation axiologique;

c. la cible5 affectée par l'avenir tel qu'il est envisagé dans l'occurrence.

4. L’approche développée par Alice Krieg-Planque (2009) sur la notion de formule aurait également pu être entreprise à partir de nos données.

5. Le mot cible réfère normalement au destinataire d'un acte délibéré. On l'utilise ici de manière plus générale, pour dénoter tout individu, collectif ou phénomène, naturel ou social, qui est représenté comme étant affecté par le changement climatique. 


\section{Résultats}

\section{Perspectives positives et négatives}

Dans un premier temps, nous nous concentrons sur les perspectives attribuées à l'avenir dans un contexte de changement climatique. Nous avons réparti les énoncés contenant les mots en question en quatre catégories : perspective négative, perspective positive, perspective réfutant la question du climat («Refus») et enfin l'absence d'attitude évaluative (« Neutre»). Le choix de ces catégories est fondé sur des recherches antérieures interrogeant de manière plus générale les conceptions des gens à propos du changement climatique (Tvinnereim et al., 2017). Les résultats sont présentés dans le tableau 1.

\begin{tabular}{l|c|c|c|c|c}
\hline & \multicolumn{5}{|c}{ Perspectives sur l'avenir en contexte climatique } \\
\hline Recherche & Négative & Positive & Refus & Neutre & Total \\
\hline 1. réchauffement - avenir 2009-2010 & 44 & 4 & 2 & 4 & 54 \\
\hline 2. avenir - réchauffement 2009-2010 & 33 & 4 & 3 & 1 & 41 \\
\hline 3. climatique - avenir 2009-2010 & 46 & 5 & 2 & 4 & 57 \\
\hline 4. avenir - climatique 2009-2010 & 25 & 4 & 0 & 9 & 38 \\
\hline 5. réchauffement - futur 2009-2010 & 14 & 3 & 0 & 5 & 22 \\
\hline 6. futur - réchauffement 2009-2010 & 29 & 3 & 4 & 8 & 44 \\
\hline 7. climatique - futur 2009-2010 & 35 & 7 & 0 & 10 & 52 \\
\hline 8. futur - climatique 2009-2010 & 42 & 2 & 0 & 26 & 70 \\
\hline TOTAL & 268 & 32 & 11 & 67 & 378 \\
\hline
\end{tabular}

\begin{tabular}{l|r|r|r|r|c}
\hline 9. réchauffement - avenir 2013-2014 & 20 & 2 & 6 & 8 & 36 \\
\hline 10. avenir - réchauffement 2013-2014 & 18 & 0 & 2 & 2 & 22 \\
\hline 11. climatique - avenir 2013-2014 & 51 & 11 & 1 & 5 & 68 \\
\hline 12. avenir - climatique 2013-2014 & 36 & 2 & 2 & 2 & 42 \\
\hline 13. réchauffement - futur 2013-2014 & 18 & 3 & 4 & 6 & 31 \\
\hline 14. futur - réchauffement 2013-2014 & 22 & 0 & 4 & 9 & 35 \\
\hline 15. climatique - futur 2013-2014 & 34 & 3 & 2 & 10 & 49 \\
\hline 16. futur - climatique 2013-2014 & 38 & 3 & 0 & 9 & 50 \\
\hline TOTAL & 237 & 24 & 21 & 51 & 333 \\
\hline
\end{tabular}

Tableau 1. Perspectives sur l'avenir en contexte climatique

Le résultat est particulièrement tranché. La grande majorité des perspectives manifestées dans les billets étudiés sont négatives : 268 occurrences sur 
un total de 378 pour la première période et 237 sur un total de 333 pour la deuxième période, soit plus des $2 / 3$ des résultats (71\%). En revanche, nous notons que les billets témoignant d'une perspective positive sur l'avenir sont relativement peu nombreux : 32 et 24 occurrences ( $8 \%$ et $7 \%$ respectivement) pour les deux périodes. Les billets n'exprimant ni perspectives positives ni perspectives négatives sont au nombre de 67 et 51 occurrences (17\% et $15 \%$ respectivement). Enfin, les billets traduisant des perspectives qui nient la question climatique sont moins importants : 11 et 21 occurrences (3\% et $6 \%$ respectivement).

Pour illustrer ces chiffres, nous présentons quelques exemples, dont les premiers représentent la grande majorité des perspectives négatives exprimées :

1. Dans le futur, le réchauffement de la planète détruira toutes les plantes vitales. (F-R-13-14)

2. Si l'on ne prend pas des mesures radicales pour adapter nos modes de vie au réchauffement, nous préparerons un monde invivable à nos enfants, pour qui l'avenir n'est déjà pas rose. (R-A-13-14)

Dans (1), c'est le verbe détruire qui contribue à la perspective négative, tandis que dans (2), deux éléments différents traduisent cette perspective : «monde invivable» et "avenir [...] pas rose».

Dans le cas des billets exprimant une perspective positive, celle-ci est traduite par le substantif chance en (3) ; à noter, toutefois, qu'il est intégré dans une question incorporant une hypothèse introduite par si :

3. Et si, en définitive, le réchauffement de la planète devenait une chance pour l'avenir de l'humanité? (R-A-09-10)

En (4), c'est la construction «élan formidable» qui permet de représenter une vision optimiste et positive de l'avenir :

4. Lutter contre le dérèglement climatique n'est pas forcément une contrainte mais plutôt un élan formidable sur lequel l'avenir peut se construire. (C-A-13-14)

La représentation «neutre » de l'avenir constitue quantitativement la deuxième catégorie, cette catégorie se référant notamment aux interrogations des scientifiques sur l'impact potentiel du changement climatique. Ces occurrences se caractérisent tant par l'absence d'expression évaluative et un ton neutre au regard de l'avenir que par l'absence de marqueurs de conflictualité, comme l'illustrent les exemples (5) et (6) :

5. La recherche scientifique sur le changement climatique examine généralement son impact dans l'avenir et comment il affectera le système physique terrestre. (C-A-13-14)

6. Et tout l'enjeu de ces découvertes, c'est aussi de connaître les effets du réchauffement climatique actuel sur notre littoral et de comprendre ce qui va se passer à l'avenir. (R-A-13-14) 
Le syntagme «théorie bidon » traduit la contestation du réchauffement climatique exprimée dans l'exemple suivant (7), classifié sous la catégorie «refus» :

7. Si nous ne parvenons pas à stopper l'avancée de la politisation et de l'institutionnalisation de la théorie bidon du réchauffement planétaire, nous connaîtrons très certainement un avenir de «science » contrôlée par décret gouvernemental et un régime mondial qui favorisera la marche de l'industrie tout en imposant des restrictions sévères et des taxes arbitraires au grand public. (R-A09-10)

La catégorie «refus» se caractérise donc par la présence de marqueurs de conflictualité ("emmerder les gens», « un avenir aussi supposé qu'incertain » dans l'exemple suivant) :

8. Adaptation au changement climatique : façon d'emmerder les gens aujourd'hui au nom d'un avenir aussi supposé qu'incertain dans une perspective apocalyptique. (C-A-09-10)

\section{La présence d'expressions évaluatives}

Dans les billets de blog analysés dans le présent travail, couvrant un total de 711 messages, nous observons une grande variation dans les expressions évaluatives, point déjà attesté par les exemples présentés dans la section ci-dessus. Dans la présente section, nous ajoutons quelques nouveaux exemples pour bien illustrer cette «subjectivation » du phénomène climatique (KerbratOrecchioni, 2009).

La grande majorité des occurrences se caractérisant par une tonalité dysphorique, ces observations viennent souligner la représentation négative de l'avenir décrite dans la section précédente. Deux scénarios se distinguent, celui de la menace et celui du risque (Petiot, 1994). Dans le premier cas, il s'agit notamment d'adjectifs épithètes évaluatifs qui contribuent à une représentation négative de l'avenir (sombre, difficile, triste) :

9. Ces déclarations ne sont pas anodines : elles font suite à la récente publication d'études qui prédisent à l'humanité un avenir sombre, d'une part à cause de la raréfaction croissante des ressources naturelles ainsi que du réchauffement climatique. (A-R-13-14)

10. Pelouses desséchées, restrictions d'eau, chaque été la rumeur enfle : les nappes d'eau souterraines seraient à sec et la France, réchauffement climatique oblige, promise à un avenir difficile. (R-A -09-10)

11. Si aucun accord pragmatique n'est pris, c'est à dire un accord digne de ce nom et prenant en compte le réchauffement climatique accéléré, alors les illuminations de la place Wilson ne seront qu'un triste avenir. (R-A-13-14)

Soulignons également la présence d'un autre groupe d'adjectifs épithètes évaluatifs qui associent l'avenir à la notion de risque, insistant sur 
les conséquences imprévisibles du changement climatique (inquiétant, chaotique) :

12. Cette méditerranée serait aussi le «laboratoire inquiétant » de l'avenir des océans si le réchauffement climatique continue ses ravages. (A-R-09-10)

13. Le réchauffement climatique nous mène inexorablement vers un avenir chaotique à moins que nous n'agissions comme une seule famille d'êtres humains. (R-A -09-10)

La présence des marqueurs mélioratifs est rare. Ces occurrences se caractérisent par une volonté d'envisager les mesures contre les changements climatiques comme une opportunité plutôt que comme une contrainte, comme dans (14), où la construction « un meilleur futur» est présentée comme cooccurrente de la limitation du changement climatique :

14. Enfin, nous avons les moyens de limiter le changement climatique en cours et construire un meilleur futur, a indiqué le secrétaire général des Nations Unies, pour qui «les décideurs doivent prendre les décisions, car le temps joue contre nous». (C-F-13-14)

Ces exemples nous donnent déjà des indications portant sur les cibles (personnes et entités affectées) de l'avenir dont on parle. Dans la section suivante, nous entreprendrons une analyse plus systématique de cette question.

\section{Un avenir pour qui ou quoi?}

Une question importante consiste à savoir ce qui est concerné par l'avenir évoqué par les blogueurs. En termes simples, quelle est la cible visée (voir note 5) par la manière d'envisager l'avenir? Pour mener à bien cette exploration, nous proposons une classification en six catégories, inspirée par les études déjà entreprises sur la conception de l'avenir dans des blogs anglais (Fløttum et al., 2014) : un avenir pour l'humanité ou les êtres humains, pour l'environnement, pour l'énergie, pour la société (dans un sens large, comprenant les aspects économiques et sociaux), pour la planète (comprenant les êtres humains aussi bien que l'environnement) et une dernière catégorie où la cible n'est pas évoquée explicitement. Nous avons déterminé les catégories de manière inductive, à travers une lecture attentive et une analyse de contenu de chaque énoncé. Ces identifications et synthèses thématiques ont été faites manuellement ${ }^{6}$. Quelques exemples relèvent de plusieurs catégories. Dans ces cas, nous avons classé le message en question en fonction de ce qui est mentionné en premier lieu. Le tableau 2 montre les résultats de cette classification.

6. Dans des corpus plus larges, la catégorisation peut se faire plus efficacement en combinant la lecture avec une catégorisation automatique, par exemple à travers l'outil «Structural Topic Modeling» (voir Tvinnereim, Fløttum, 2015). 


\begin{tabular}{|c|c|c|c|c|c|c|c|}
\hline \multicolumn{8}{|c|}{ Avenir pour qui/quoi? } \\
\hline Recherche & Humanité & $\begin{array}{l}\text { Environ- } \\
\text { nement }\end{array}$ & Société & Énergie & Planète & $\begin{array}{l}\text { Non } \\
\text { spécifié } \\
\text { /Autre }\end{array}$ & Total \\
\hline \multicolumn{8}{|l|}{$2009-2010$} \\
\hline \multicolumn{8}{|l|}{ Avenir } \\
\hline 1 & 19 & 8 & 6 & 2 & 10 & 9 & 54 \\
\hline 2 & 6 & 6 & 8 & 1 & 12 & 8 & 41 \\
\hline 3 & 6 & 5 & 10 & 5 & 9 & 22 & 57 \\
\hline 4 & 3 & 7 & 10 & 6 & 3 & 9 & 38 \\
\hline \multicolumn{8}{|l|}{ Futur } \\
\hline 5 & 5 & 5 & 7 & 1 & 3 & 1 & 22 \\
\hline 6 & 4 & 13 & 11 & 6 & 7 & 2 & 44 \\
\hline 7 & 8 & 6 & 11 & 6 & 5 & 16 & 52 \\
\hline 8 & 8 & 14 & 37 & 3 & 3 & 5 & 70 \\
\hline Total & 59 & 64 & 100 & 30 & 52 & 72 & 377 \\
\hline \multicolumn{8}{|l|}{ 2013-2014 } \\
\hline \multicolumn{8}{|l|}{ Avenir } \\
\hline 9 & 12 & 5 & 3 & 2 & 4 & 10 & 36 \\
\hline 10 & 3 & 2 & 3 & 2 & 5 & 7 & 22 \\
\hline 11 & 11 & 11 & 17 & 4 & 9 & 16 & 68 \\
\hline 12 & 4 & 6 & 10 & 7 & 0 & 15 & 42 \\
\hline \multicolumn{8}{|l|}{ Futur } \\
\hline 13 & 0 & 6 & 11 & 0 & 5 & 9 & 31 \\
\hline 14 & 4 & 13 & 11 & 1 & 5 & 1 & 35 \\
\hline 15 & 11 & 4 & 22 & 2 & 3 & 7 & 49 \\
\hline 16 & 9 & 11 & 20 & 4 & 1 & 5 & 50 \\
\hline Total & 54 & 58 & 97 & 22 & 32 & 70 & 333 \\
\hline
\end{tabular}

Tableau 2. Avenir pour qui/quoi?

Nous observons d'abord qu'il y a une grande variation dans les cibles concernées et dans la manière d'envisager l'avenir, qui sera perçu comme plus ou moins modifié par le changement climatique. Pour les deux périodes, c'est la perspective économique et sociale qui est le plus souvent mentionnée dans les messages étudiés, mais les autres perspectives sont également bien représentées, bien qu'un peu moins pour la perspective énergétique.

La société est donc quantitativement la première catégorie affectée dans le corpus, et les conséquences sont considérées sous de multiples facettes. 
Il s’agit soit des conséquences pour la société en général, comme dans l'exemple (15), soit d'aspects spécifiques : «le développement économique et social» dans l'exemple (16), les « réfugiés climatiques» dans l'exemple (17).

15. Sortons un peu de l'actualité immédiate pour réfléchir sur l'avenir de nos sociétés face aux menaces environnementales qui nous guettent, qu'elles soient d'ordre climatique ou en termes de perte de biodiversité. (A-C-09-10)

16. Dans l'avenir, avec le réchauffement climatique, les situations d'évènements extrêmes comme les sécheresses, les inondations et l'érosion accélérée, risquent de se multiplier et d'être, avec la pollution et le gaspillage, d'ici une seule génération un des principaux facteurs limitant le développement économique et social dans la plupart des pays du monde. (A-R-09-10)

17. Dans un avenir de plus en plus proche, le réchauffement climatique affectera durablement la production alimentaire, tandis que le nombre des réfugiés climatiques ne cessera d'augmenter. (A-R-13-14)

La catégorie que nous avons étiquetée «environnement» regroupe divers aspects des conséquences environnementales du changement climatique, comme les effets sur les espèces sauvages (18), les océans (19) ou la biodiversité (20):

18. D'autant que le réchauffement pourrait à l'avenir toucher gravement un plus grand nombre d'espèces sauvages, selon l'UICN. (R-A-09-10)

19. Cette méditerranée serait aussi le laboratoire inquiétant de l'avenir des océans si le réchauffement climatique continue ses ravages selon Pierre Chevaldonné, chercheur du CNRS au Centre d'Océanologie de Marseille. (A-R-09-10)

20. La situation ne devrait d'ailleurs pas aller en s'arrangeant puisque le changement climatique accélérera à l'avenir le rythme des extinctions, estiment les chercheurs. «L'état général de la biodiversité s'aggrave, dans de nombreux cas de manière significative» indique dans le rapport Derek Tittensor, écologiste marin au Centre de surveillance de la conservation de la nature. (C-A-13-14)

La troisième catégorie réfère à l'humanité, réalisée par des lexèmes comme l'humanité (21), ou par des syntagmes comme générations futures (22) ou êtres humains (23):

21. La "guerre climatique » est susceptible de menacer l'avenir de l'humanité, mais elle a été exclue des rapports du GIEC, qui a reçu le Prix Nobel de la Paix en 2007. (C-A-09-10)

22. En effet, les mots et les déclarations d'intention ne suffisent plus, il importe d'agir vite contre le réchauffement global et le changement climatique pour l'avenir des générations futures. (R-A-09-10)

23. Le réchauffement climatique nous mène inexorablement vers un avenir chaotique à moins que nous n'agissions comme une seule famille d'êtres humains. (R-A-09-10) 
La quatrième catégorie concerne la planète ou la Terre, et les occurrences soulignent la vulnérabilité de celle-ci face au changement climatique. Nous sommes donc dans une perspective de menace et de risque.

24. Le réchauffement du climat, depuis la fin du xixe siècle, dessine un avenir inquiétant pour la planète et les générations futures. (R-A-09-10)

25. [...] nous ne pourrons collectivement gagner le combat climatique que si chaque habitant de notre irremplaçable planète Terre, présent et futur, y est impliqué. Ce combat sera très coûteux [...]. (C-F-13-14)

26. Le changement climatique est un élément central dans la crise systémique du capitalisme, il menace l'avenir de la vie sur la planète et met clairement en lumière l'incapacité du modèle actuel à le résoudre réellement. (C-A-13-14)

Cette catégorie se caractérise aussi par une tonalité émotionnelle, presque pathétique, comme dans (27):

27. Au jour d'aujourd'hui, notre Mère-Terre est blessée et le futur de l'humanité est en danger. (F-R-09-10)

L'énergie constitue la catégorie la moins importante quantitativement. Les mesures contre le changement climatique y sont mises en rapport avec la politique énergétique et les énergies renouvelables :

28. Les problématiques relatives à notre avenir énergétique et à la lutte contre le réchauffement climatique sont des sujets complexes. (A-R-13-14)

29. Plus on exploite les ressources finies, moins on investit dans les énergies d'avenir et plus on compromet le futur de la planète, déjà mal en point avec le réchauffement climatique. (F-R-13-14)

30. Or, on sait que le charbon constituera la grande menace climatique de l'avenir, au moins pour la seconde moitié du siècle quand pétrole et gaz seront par la force des choses réduits à un rôle marginal (autrement dit, il n'y en aura plus). (C-A-09-10)

31. Il s'agit de préparer dès aujourd'hui un futur moins gourmand en énergie en élaborant des systèmes de production, de consommation et de vie en commun conciliables avec les contraintes écologiques, économiques et sociales qui s'annoncent. (C-F-09-10)

Dans de nombreux cas, il n'est pas possible de restituer l'entité spécifique qui serait affectée par le changement climatique, c'est l'avenir plus généralement qui est envisagé, notamment dans une perspective négative :

32. Cette augmentation de la concentration serait responsable de la tendance au réchauffement climatique déjà observée, et pourrait avoir dans l'avenir des conséquences beaucoup plus dramatiques si aucune mesure n'est prise. (R-A-09-10)

33. Si nous perdons cette décennie, c'est le chaos climatique assuré. Aucun futur accord ne sera en mesure de réparer les dégâts occasionnés [...]. (C-F-13-14) 


\section{Discussion}

À travers les analyses des représentations de l'avenir telles qu'elles sont formulées dans un large corpus de blogs liés aux changements climatiques et écologiques, nous avons tenté, dans cet article, de développer une approche principalement lexicale et qualitative, soutenue par des retours au contexte discursif portant sur l'environnement. Notre but a été de contribuer à une meilleure compréhension de la manière dont est envisagé l'avenir. Nous voulions déterminer si l'avenir est perçu de manière positive ou négative, et quelles sont les cibles (individus, collectifs, société, nature) affectées par le changement climatique les plus mentionnées.

Pour reprendre nos questions de recherche, nous avons pu observer que, premièrement, les perspectives négatives et pessimistes dominent grandement sur les perspectives positives et optimistes, dans les deux périodes prises en compte. Deuxièmement, la forte présence de lexèmes évaluatifs corrobore cette tendance. Nous savons que les opinions et les attitudes portant sur la question du climat varient beaucoup selon les intérêts, les valeurs et les préférences politiques et que les débats environnementaux sont un lieu d'intense conflictualité. Cela peut indiquer que la communication portant sur le changement climatique représente avant tout un discours alarmiste, qui inquiète, et qui peut même s'orienter vers une perspective de «fin du monde » (Fløttum et al., à paraître). Cette observation rejoint celle de G. Petiot (1994), qui remarque la présence du thème de la menace et du risque dans les discours sur l'écologie.

Du nombre modeste de perspectives positives et optimistes, nous pouvons déduire que les possibilités et opportunités généralement liées à la notion de "société durable» sont peu discutées. Un nombre croissant de recherches en psychologie montre qu'un accent mis sur les opportunités pourrait contribuer à un engagement plus important des citoyens (Stoknes, 2015). Nous pensons que la manière de présenter linguistiquement le phénomène complexe du changement climatique a une influence décisive sur l'engagement des citoyens dans la cause climatique.

Nos résultats corroborent aussi quatre études publiées en 2012 dans un numéro de Communication \& langages intitulé Discours surle changement climatique et jeunesse. Ces études portent sur le discours climatique dans des documentaires télévisés (Bolka-Tabary, 2012), des ouvrages documentaires (Kovacs, 2012), des romans (Fodor, 2012), et des publications périodiques de vulgarisation scientifique (Micheau, 2012). La représentation du changement climatique dans ces différents genres se caractérise principalement par l'angoisse, les sentiments de menace et de catastrophe face à l'avenir.

Or, si les textes francophones s'avèrent largement dysphoriques à propos du climat, on note un contraste avec des blogs anglophones, où l'on trouve 
davantage de représentations positives de l'avenir construites autour de la notion de sustainability, et ce notamment dans le domaine économique (Fløttum et al., 2014).

Troisièmement, nous avons pu montrer que, lorsque les blogueurs parlent de l'avenir, c'est un avenir concernant diverses entités : notamment la société, l'environnement, les êtres humains et la planète. Nous pensons que ces résultats montrent combien la question du réchauffement climatique est complexe, tant sur le plan des causes que des conséquences, mais aussi des possibles solutions pour en atténuer les effets négatifs.

Notre quatrième interrogation portait sur les différences potentielles entre les années 2009-2010 et les années 2013-2014. La différence est insignifiante, marquée par une légère baisse des occurrences de la première à la seconde période étudiée (378 occurrences pour la période 2009-2010 contre 333 occurrences pour la période 2013-2014, voir tableau 1). C'est là un résultat qui pourra surprendre. En effet, après la COP 15 de Copenhague en 2009, les recherches climatiques n'ont cessé de souligner la gravité des changements climatiques dans les différentes régions du monde. Le GIEC, dont l'un des rôles est de résumer ces recherches, a publié en 2013-2014 les rapports AR5, très explicites quant aux effets dramatiques des activités humaines sur le climat (Fløttum et al., 2016). En outre, la perspective de la COP 21 tenue à Paris en 2015 pourrait laisser supposer que la question climatique avait fait l'objet de débats dans la blogosphère française. Au regard du contexte, on peut en effet s'étonner de ce que les blogueurs soient moins actifs en 2013-2014 qu'en 2009-2010. Il est possible que la baisse soit due, après des années de pronostics et d'avertissements, à une «fatigue climatique ». Il est également possible que l'accident nucléaire à Fukushima au Japon à la suite du tsunami de 2011 ait contribué à attirer l'attention vers les risques de l'énergie nucléaire, au détriment de la problématique climatique.

Nos résultats constituent de nouvelles connaissances à propos des dimensions humaines mais aussi sociétales des questions se présentant dans les contextes climatique et environnemental. Nous avons pu accéder à diverses conceptions des conséquences du changement climatique, conséquences clairement envisagées en termes de risques et de menaces dans le présent corpus. Le nombre de messages positifs, évoquant de possibles solutions dans lesquelles le grand public pourrait s'engager, est modeste. Bien que le cadre de cette étude soit restreint, elle permet la production de connaissances qui nous semblent essentielles pour les agendas des politiciens et décideurs. L'analyse des discours traitant du réchauffement climatique peut se montrer fortement opératoire pour travailler à l'engagement des citoyens et pour proposer et faire adopter des mesures spécifiques permettant de faire face aux défis qui s'annoncent. 


\section{Références}

ANTHONY Laurence, 2005, «AntConc: design and development of a freeware corpus analysis toolkit for the technical writing classroom », dans 2005 IEEE International Professional Communication Conference Proceedings, Piscataway (New Jersey), Institute of Electrical and Electronics Engineers, p. 729-737.

BOLKA-TABARY Laure, 2012, "Le changement climatique à la télévision : de la science à la fiction », Communication \& langages, nº 172, p. 53-67.

Сомву Jean-Baptiste, 2015, La question climatique : genèse et dépolitisation d'un problème public, Paris, Raisons d'agir.

FLøTTUm Kjersti éd., 2017, The Role of Language in the Climate Change Debate, New York, Londres, Routledge.

- 2016, "Linguistic analysis in climate change communication », dans Oxford Research Encyclopedia of Climate Science, Oxford, Oxford University Press, http://climatescience.oxfordre.com/view/10.1093/acrefore/9780190228620.001.0001/acrefore9780190228620-e-488 (consulté le 8 novembre 2018).

FLøTTUM Kjersti, DAHLTrine, 2014, «IPCC communicative practices: A linguistic comparison of the Summary for Policymakers 2007 and 2013 ", LSP Journal, vol. V, nº 2, p. 66-83.

- 2012, "Different contexts, different "stories"? A linguistic comparison of two development reports on climate change», Language \& Communication, vol. XXXII, nº 1 , p. 14-23.

FLøTTUM Kjersti, GASPER Des, ST. CLAIR Asunción Lera, 2016, «Synthesizing a policy-relevant perspective from the three IPCC "Worlds" - A comparison of topics and frames in the SPMs of the Fifth Assessment Report ", Global Environmental Change, n 38 , p. 118-129.

FLøTTUM Kjersti, GJERSTAD Øyvind, 2016, « Narratives in climate change discourse », WIRES Climate Change, vol. VIII, $\mathrm{n}^{0} 1$, https://onlinelibrary.wiley.com/doi/full/10.1002/ wcc.429 (consulté le 8 novembre 2018).

FLøTTUm Kjersti, GJeRSTAD Øyvind, TVInnEREIM Endre, à paraître, «Associations évoquées par le changement climatique chez des citoyens français et norvégiens », Bergen Language and Linguistic Studies (BeLLS).

FLøtTum Kjersti, GjesdalAnje Müller, GJeRSTAd Øyvind, Koteyko Nelya, Salway Andrew, 2014, "Representations of the future in English language blogs on climate change », Global Environmental Change, n² 29, p. 213-222.

FOdor Ferenc, 2012, «Les jeunes face au changement climatique dans l'imaginaire romanesque », Communication \& langages, n 172 , p. 83-95.

GJESDAL Anje Müller, GJERSTAD Øyvind, 2015, «Web 2.0 et genres discursifs : l'exemple de blogs sur le changement du climat», Synergies Pays Scandinaves, nº 9, p. 49-61.

GRUNDMANN Reiner, KRISHNAMURTHY Ramesh, 2010, «The discourse of climate change: A corpus-based approach », Critical Approaches to Discourse Analysis across Disciplines, vol. IV, n² 2, p.125-146.

HuLme Mike, 2009, Why We Disagree about Climate Change: Understanding Controversy, Inaction and Opportunity, Cambridge, Cambridge University Press.

Kerbrat-ORECCHIONI Catherine, 2009, L'énonciation: de la subjectivité dans le langage, Paris, Armand Colin.

КотеYко Nelya, 2010, «Mining the internet for linguistic and social data: An analysis of “carbon compounds" in Web feeds », Discourse \& Society, vol. XXI, nº 6, p. 655-674. 
Kovacs Susan, 2012, «Engager et enrôler les jeunes dans la lutte contre le changement climatique : le documentaire jeunesse et l'attitude des collégiens d'aujourd'hui », Communication \& langages, no 172 , p. 69-81.

KRIEG-PLANQUE Alice, 2009, La notion de "formule » en analyse du discours : cadre théorique et méthodologique, Besançon, Presses universitaires de Franche-Comté.

Mauger-Parat Marion, Peliz Ana Carolina, 2013, «Controverse, polémique, expertise : trois notions pour aborder le débat sur le changement climatique en France», [VertigOJ. La revue électronique en sciences de l'environnement, vol. XIII, $\mathrm{n}^{\circ} 2$.

MeURER Paul, 2012, "Corpuscle - a new corpus management platform for annotated corpora », dans Exploring Newspaper Language: Using the Web to Create and Investigate a Large Corpus of Modern Norwegian, G. Andersen éd., Amsterdam, John Benjamins Publishing Company, p. 29-50.

MiCHEAU Beatrice, 2012, «Le changement climatique dans la presse magazine : expliquer la menace, impliquer les individus, prédire la catastrophe», Communication \& langages, $\mathrm{n}^{\circ} 172, \mathrm{p} .27-51$.

Moser Susanne C., 2016, «Reflections on climate change communication research and practice in the second decade of the 21st century: What more is there to say? », WIRES Climate Change, vol. VII, n³ 3 , https://onlinelibrary.wiley.com/doi/full/10.1002/ wcc.403 (consulté le 8 novembre 2018).

NTAP French corpus, hébergé par le Clarino Bergen Center, https://clarin.w.uib.no/ (consulté le 8 novembre 2018).

PAINTER James, 2013, Climate Change in the Media: Reporting Risk and Uncertainty, Londres, I. B. Tauris.

Pearce Warren, Brown Brian, Nerlich Brigitte et al., 2015, "Communicating climate change: conduits, content, and consensus », WIREs Climate Change, vol. VI, nº 6 , p. 613-626.

Petiot Geneviève, 1994, "Les mots de l'écologie", Mots. Les langages du politique, n०39, p. 69-78.

SAlway Andrew, ElgeSEm Dag, HofLAnd Knut et al., 2016, «Topically-focused blog corpora for multiple languages», dans Proceedings of the 1oth Web as Corpus Workshop, Berlin, Association for Computational Linguistics, p. 17-26.

SCHÄFER Mike S., SCHLICHTING Inga, 2014, «Media representations of climate change: A meta-study of the research field », Environmental Communication, vol. VIII, n², p. $142-160$.

Sto knes Per Eespen, 2015, What We Think About When We Try Not to Think About Global Warming: Toward a New Psychology of Climate Action, White River Junction (Vermont), Chelsea Green Publishing.

TVINNEREIM Endre, FLøTTUM Kjersti, 2015, «Explaining topic prevalence in answers to open-ended survey questions about climate change », Nature Climate Change, n ${ }^{\circ} 5$, p. 744-747.

TVInNEReIm Endre, FLøTtum Kjersti, GJeRSTAD Øyvind et al., 2017, «Citizens’ preferences for tackling climate change. Quantitative and qualitative analyses of their freely formulated solutions », Global Environmental Change, n 46, p. 34-41.

Zaccal Edwin, Gemenne François, Decroly Jean-Michel éd., 2012, Controverses climatiques, sciences et politique, Paris, Presses de Sciences Po. 


\section{Résumé / Abstract / Compendio}

\section{Avenir et climat : représentations de l'avenir dans des blogs francophones portant sur le changement climatique}

Cet article explore la manière dont l'avenir est perçu dans des blogs portant sur les changements climatiques, extraits du corpus NTAP (Networks of Text and People). L'analyse, principalement entreprise dans une perspective lexicale, se concentre sur les périodes 2009-2010 et 2013-2014. Les résultats montrent que les perspectives négatives et pessimistes dominent grandement sur les perspectives positives et optimistes.

Mots-clés : changement climatique, réchauffement, avenir, langage évaluatif, blogs

\section{Future and climate: representations of the future in French language blogs related to climate change}

This article investigates how the future is perceived in blogs related to climate change, extracted from the corpus NTAP (Networks of Text and People). The analysis, mainly undertaken in a lexical perspective, focuses on the periods 2009-2010 and 2013-2014. The results show that the negative and pessimistic perspectives are clearly more prevalent than the positive and optimistic ones.

Keywords: climate change, warming, future, evaluative language, blogs

\section{Porvenir y clima: representaciones del futuro en blogs francófonos sobre el tema del cambio climático}

Este artículo explora la manera con la cual el porvenir está percibido en blogs sobre los cambios climáticos sacados del corpus NTAP (Networks of Text and People). El análisis, principalemente orientado dentro de una perspectiva lexical, se concentra sobre los periódos 2009-2010 y 2013-2014. Los resultados muestran que las perspectivas negativas y pesimistas dominan fuertemente las perspectivas positivas y optimistas.

Palabras claves: cambio climatico, calentamiento, porvenir, lenguaje evaluador, blogs 